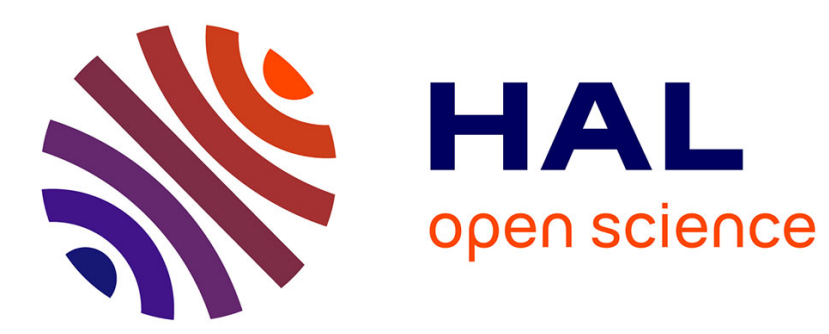

\title{
Miroir pour l'examen d'un objet cylindrique sous une incidence constante le long d'hélices données
}

\author{
J. Perdijon
}

\section{To cite this version:}

J. Perdijon. Miroir pour l'examen d'un objet cylindrique sous une incidence constante le long d'hélices données. Revue de Physique Appliquée, 1975, 10 (5), pp.319-324. 10.1051/rphysap:01975001005031900 . jpa-00243919

\section{HAL Id: jpa-00243919 https://hal.science/jpa-00243919}

Submitted on 1 Jan 1975

HAL is a multi-disciplinary open access archive for the deposit and dissemination of scientific research documents, whether they are published or not. The documents may come from teaching and research institutions in France or abroad, or from public or private research centers.
L'archive ouverte pluridisciplinaire HAL, est destinée au dépôt et à la diffusion de documents scientifiques de niveau recherche, publiés ou non, émanant des établissements d'enseignement et de recherche français ou étrangers, des laboratoires publics ou privés. 
Classification

Physics Abstracts

$0.610-0.640-9.170$

\title{
MIROIR POUR L'EXAMEN D'UN OBJET CYLINDRIQUE SOUS UNE INCIDENCE CONSTANTE LE LONG D'HÉLICES DONNÉES
}

\author{
J. PERDIJON \\ Centre d'Etudes Nucléaires, 38041 Grenoble, France
}

(Reçu le 12 février 1975, accepté le 24 avril 1975)

\begin{abstract}
Résumé. - Quelle surface doit avoir un miroir pour transformer des ondes planes, se propageant parallèlement aux génératrices d'un objet cylindrique, en ondes frappant le cylindre sous une incidence constante le long d'hélices données ? On a obtenu une surface réglée développable que l'on a précisée quand le cylindre était prismatique ou de révolution et quand les hélices étaient des génératrices ou des directrices planes du cylindre. Il est ainsi possible d'examiner totalement l'objet par une simple translation. Une première application est le contrôle des tubes par ultrasons sans mise en rotation.
\end{abstract}

\begin{abstract}
What is the surface of a mirror which transforms plane waves propagating parallel to the generators of a cylindrical object into waves striking the cylinder with the same incidence along given helices ? We obtained a developable surface and equations are given when the cylinder is prismatic or of revolution and when the helices are generators or plane directrices of the cylinder. So, it is possible to examine the whole object only by translation. One application is the ultrasonic testing of tubes without rotating them.
\end{abstract}

1. Introduction. - On dispose d'une source d'ondes planes, de nature mécanique ou électromagnétique, et on désire utiliser son rayonnement pour examiner toute la surface d'un objet cylindrique par simple translation de celui-ci relativement à la source.

Afin que, lors de la translation, tous les points de l'objet soient également examinés, quelle que soit leur longitude $\left({ }^{1}\right)$, on place la source de façon qu'elle émette parallèlement aux génératrices ; on évite ainsi la formation de zones d'ombre.

L'examen d'un objet repose sur la variation de l'intensité des ondes réfléchies ou diffractées par l'objet ou bien réfractées dans l'objet. Afin de pouvoir comparer les intensités obtenues pour chaque point de l'objet, les ondes incidentes doivent avoir une direction fixe par rapport aux normales et aux génératrices.

Lorsque l'examen se fait par écho (cas habituel des contrôles par ultrasons), on choisit cette direction fixe de telle façon que les rayons réfractés soient aussi voisins que possible de la congruence des normales aux surfaces des discontinuités que l'on veut détecter; on obtient ainsi un écho d'amplitude maximale. Or, les

(1) Par commodité d'expression, on étendra ici au cylindre la définition de certains termes (longitude, déclinaison, méridien, équatorial) dont l'usage est habituellement limité au cas de la sphère. discontinuités que l'on recherche ont souvent une direction préférentielle par rapport à la surface de l'objet. C'est ainsi que, pour des tubes sans soudure, les défauts sont principalement contenus dans des plans parallèles (défauts longitudinaux) ou perpendiculaires (défauts transversaux) à l'axe ; pour des tubes soudés en hélice, ils sont dans des plans normaux également inclinés par rapport à l'axe. On cherchera donc à engendrer dans l'objet des rayons formant une congruence de droites également inclinées de l'angle $r$ par rapport aux normales et également inclinées de l'angle $\beta$ par rapport aux génératrices.

2. But du miroir cherché. - On sait que, par réflexion ou par réfraction, une congruence de droites est changée en une autre congruence de droites (théorème de Malus et Dupin).

Soit (C) un objet cylindrique (voir Fig. 1). En un point $A$ de cet objet, on veut que le rayon réfracté $A Q$ soit incliné de $r$ par rapport à la normale et de $\beta$ par rapport aux génératrices. Le rayon incident correspondant $M A$ doit donc appartenir à une congruence de droites telles que chacune est inclinée de :

- $i$ (angle d'incidence) par rapport à la normale en $A$ à $(\mathrm{C})$; si on désigne par $n$ l'indice relatif de l'objet, on a :

$$
\sin i=n \sin r
$$




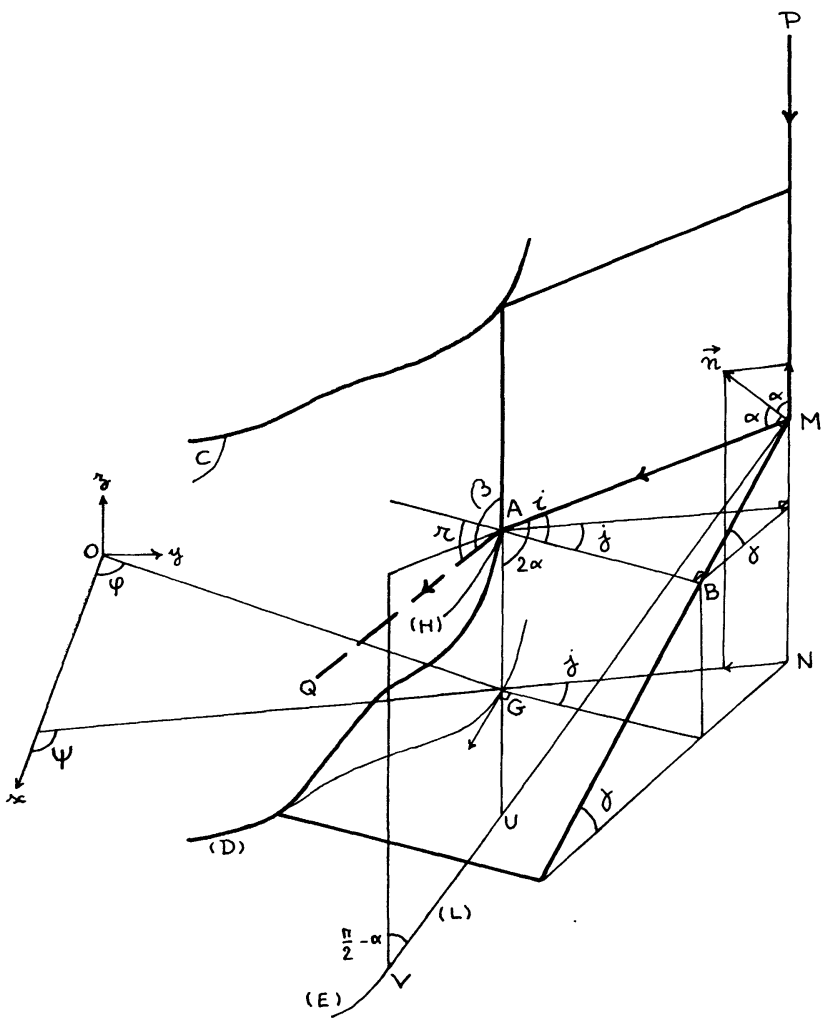

Fig. 1. - Cas général.

- $2 \alpha$ (angle complémentaire de la déclinaison) par rapport à la génératrice de $(\mathrm{C})$ passant par $A$; on montre que l'on a :

$$
\cos 2 \alpha=n \cos \beta \text {. }
$$

Soit $\mathrm{PN}$ un rayon émis parallèlement aux génératrices de $(C)$. On va chercher à déterminer quelle surface (S) de miroir il faut interposer dans la congruence des rayons parallèles tels que $P N$ pour obtenir, après réflexion, une congruence de rayons incidents sur (C) tels que $M A$.

On délimitera ensuite cette surface (S) de façon que l'amplitude de l'onde réfractée soit indépendante de la longitude du point examiné $Q$.

3. Mise en équation (axes orthogonaux). - Soit le cylindre $(\mathrm{C})$, de génératrices parallèles à $O z$, de directrice dans le plan $x O y$ une courbe fermée (D) (Fig. 1). Un point $G$ de (D) a pour coordonnées, en fonction du paramètre $\varphi=(O x, O G)$ :

$$
X=f(\varphi), \quad Y=g(\varphi) .
$$

Soit $z(x, y)$ l'équation de la surface (S) du miroir cherché. La normale $\mathbf{n}$ à cette surface est contenue dans le plan $P M A$ parallèle à $O z$. Ce plan fait un angle constant $j$ avec le plan méridien $B A G$, plan contenant la normale $A B$ et la génératrice $A G$, et l'on a :

$$
\cos j=\frac{\cos i}{\sin 2 \frac{\alpha}{}}
$$

La surface (S) peut donc être engendrée par une courbe (L) contenue dans le plan $P M A$, d'équation :

$$
[x-f(\varphi)] \sin \psi-[y-g(\varphi)] \cos \psi=0
$$

avec :

$$
\operatorname{tg} \psi=\frac{-f^{\prime}(\varphi)+g^{\prime}(\varphi) \operatorname{tg} j}{f^{\prime}(\varphi) \operatorname{tg} j+g^{\prime}(\varphi)}
$$

De plus, $\mathbf{n}$ doit être bissectrice de l'angle constant $\widehat{P M A}=2 \alpha$. La courbe (L) est donc une droite faisant l'angle $\left(\frac{\pi}{2}-\alpha\right)$ avec $O z$ et son équation est :

$$
\left\{\begin{array}{l}
z \operatorname{cotg} \alpha=x \cos \psi+y \sin \psi+\lambda(\psi) \\
x \sin \psi-y \cos \psi=f(\varphi) \sin \psi-g(\varphi) \cos \psi .
\end{array}\right.
$$

Le plan tangent étant le même le long d'une génératrice (L), (S) est développable, d'où :

$$
\lambda^{\prime}(\psi)=f(\varphi) \sin \psi-g(\varphi) \cos \psi .
$$

La surface (S) admet pour arête de rebroussement la courbe (E) dont un point $V$ a pour coordonnées :

$$
\left\{\begin{array}{l}
x=\lambda^{\prime}(\psi) \sin \psi+\lambda^{\prime \prime}(\psi) \cos \psi \\
y=-\lambda^{\prime}(\psi) \cos \psi+\lambda^{\prime \prime}(\psi) \sin \psi \\
z=\left[\lambda(\psi)+\lambda^{\prime \prime}(\psi)\right] \operatorname{tg} \alpha .
\end{array}\right.
$$

C'est une hélice dont on désignera le pas par $p$ et qui est tracée sur le cylindre $(\Gamma)$, d'équation en coordonnées cylindriques :

$$
\rho^{2}=\lambda^{\prime 2}(\psi)+\lambda^{\prime \prime 2}(\psi) .
$$

Dans les mêmes coordonnées, l'équation de (S) s'écrit :

$$
\rho^{2}=[z \operatorname{cotg} \alpha-\lambda(\psi)]^{2}+\lambda^{\prime 2}(\psi) .
$$

4. Résolution. - La surface (S) du miroir cherché est donc donnée par les éq. (6) et (4). On va calculer $\lambda$ dans deux cas qui sont d'ailleurs les plus courants.

4.1 Cylindre Prismatique RÉGUlier. - Soit le cylindre (C) d'axe $O z$ et de directrice (D) un polygone régulier à $m$ côtés de longueur $a$. Plaçons la première face telle que $x O z$ en soit le plan médiateur. D'après (5), on a pour cette face $\psi=j$, donc $\lambda(\psi)$ est une constante $c_{1}$. La surface du miroir correspondant est un plan, d'équation :

$$
z \operatorname{cotg} \alpha=x \cos j+y \sin j+c_{1} .
$$

Considérons la face se déduisant de la précédente par une rotation de $-2 \pi / m$ autour de $O z$. Le miroir plan qui lui est associé a pour équation :

$$
z \operatorname{cotg} \alpha=x \cos \left(j-\frac{2 \pi}{m}\right)+y \sin \left(j-\frac{2 \pi}{m}\right)+c_{2} \text {. }
$$

Si on veut avoir continuité de phase d'une face à l'autre, il faut que les trajets des ondes incidentes éclai- 
rant un point donné de l'arête entre les deux faces soient égaux pour les deux faces. En prenant $c_{1}=c_{2}$, l'onde provenant du premier miroir serait retardée de $a \sin j$ par rapport à celle provenant du second miroir. On choisira donc :

$$
c_{2}=c_{1}-a \sin j .
$$

Les équations des miroirs associés aux autres faces se déduisent de (12) et (13). L'ensemble (S) de ces miroirs forme une sorte de surface hélicoïdale à facettes, de pas:

$$
p=m a \sin j \cdot \operatorname{tg} \alpha .
$$

On a représenté deux surfaces (S) obtenues pour un prisme hexagonal avec $i=30^{\circ}$ :

- sur la figure 2 , on a choisi :

$$
2 \alpha=\frac{\pi}{2} \pm i
$$

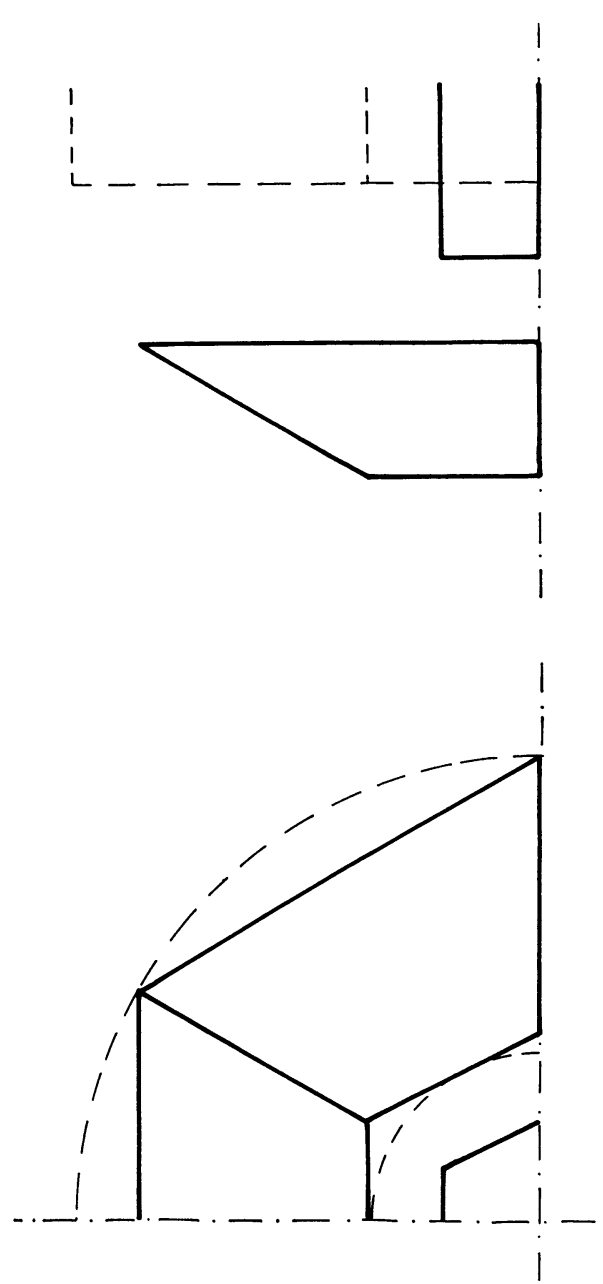

Fig. 2. - Miroir pour la détection des défauts transversaux dans un tube hexagonal.

ce qui fait que le rayon émis $P M$, le rayon incident $M A$ et le rayon réfracté $A Q$ sont tous dans un même plan méridien du cylindre $(j=0$, cas de la détection des défauts transversaux). La surface du miroir est un tronc de pyramide régulier à six faces $(p=0)$, d'angle au sommet :

$$
2 \delta=\pi-2 \alpha=\frac{\pi}{2} \mp i
$$

- sur la figure 3 , on a choisi :

$$
2 \alpha=\frac{\pi}{2}
$$
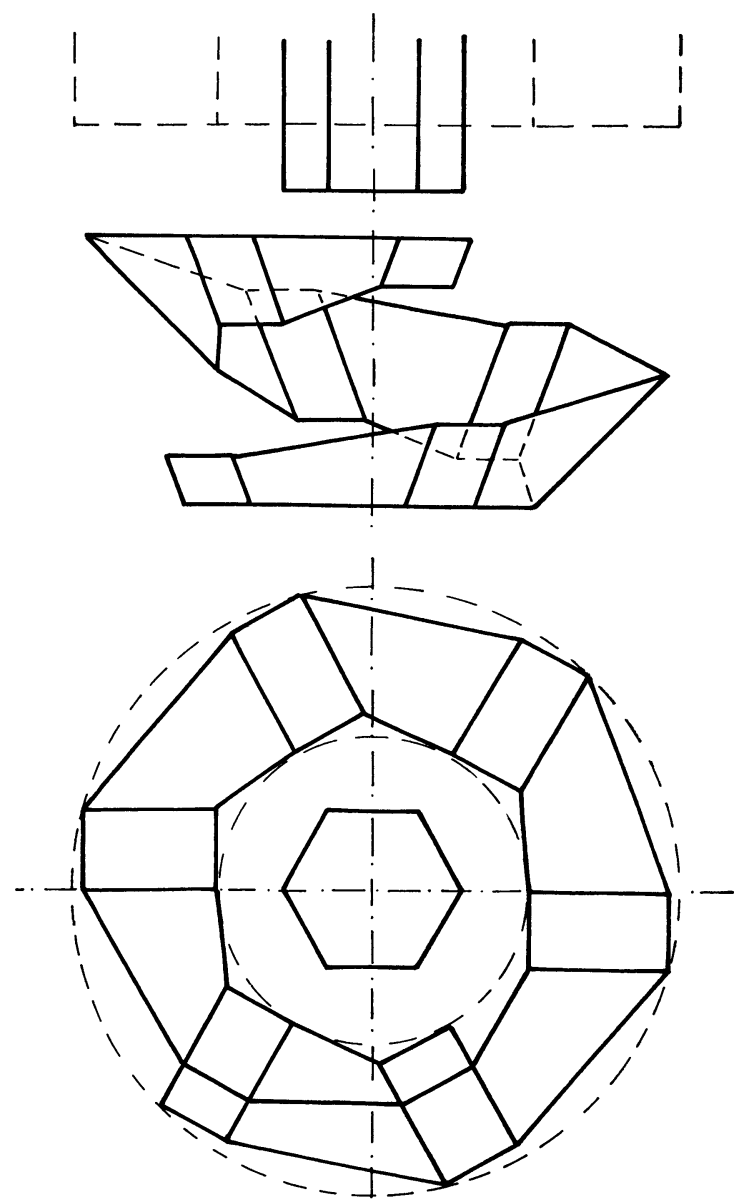

Fig. 3. - Miroir pour la détection des défauts longitudinaux dans un tube hexagonal.

ce qui fait que le rayon incident $M A$ et le rayon réfracté $A Q$ sont dans un même plan équatorial du cylindre $(j=i$, cas de la détection des défauts longitudinaux). Le miroir peut être obtenu par pliage.

4.2 CylindRe DE RÉvolution. - Soit le cylindre (C) d'axe $O z$ et de directrice (D) un cercle de rayon $R$. D'après (5) et (7), on a $\psi=\varphi+j$ et $\lambda^{\prime}(\psi)=R \sin j$. La génératrice $(\mathrm{L})$ du miroir (S) a pour équation :

$$
\left\{\begin{aligned}
z \operatorname{cotg} \alpha & =x \cos \psi+y \sin \psi+\psi \cdot R \sin j \\
R \sin j & =x \sin \psi-y \cos \psi .
\end{aligned}\right.
$$

La surface (S) admet pour arête de rebroussement une hélice circulaire de pas :

$$
p=2 \pi R \sin j \cdot \operatorname{tg} \alpha .
$$


Son équation en coordonnées cylindriques s'écrit, d'après (10) :

$$
\rho^{2}=(z \operatorname{cotg} \alpha-\psi \cdot R \sin j)^{2}+R^{2} \sin ^{2} j .
$$

On a représenté deux surfaces (S) correspondant à $i=30^{\circ}$ :

- sur la figure 4, $\alpha$ est donné par la relation (15). La surface du miroir est un tronc de cône, d'angle au sommet donné par (16),

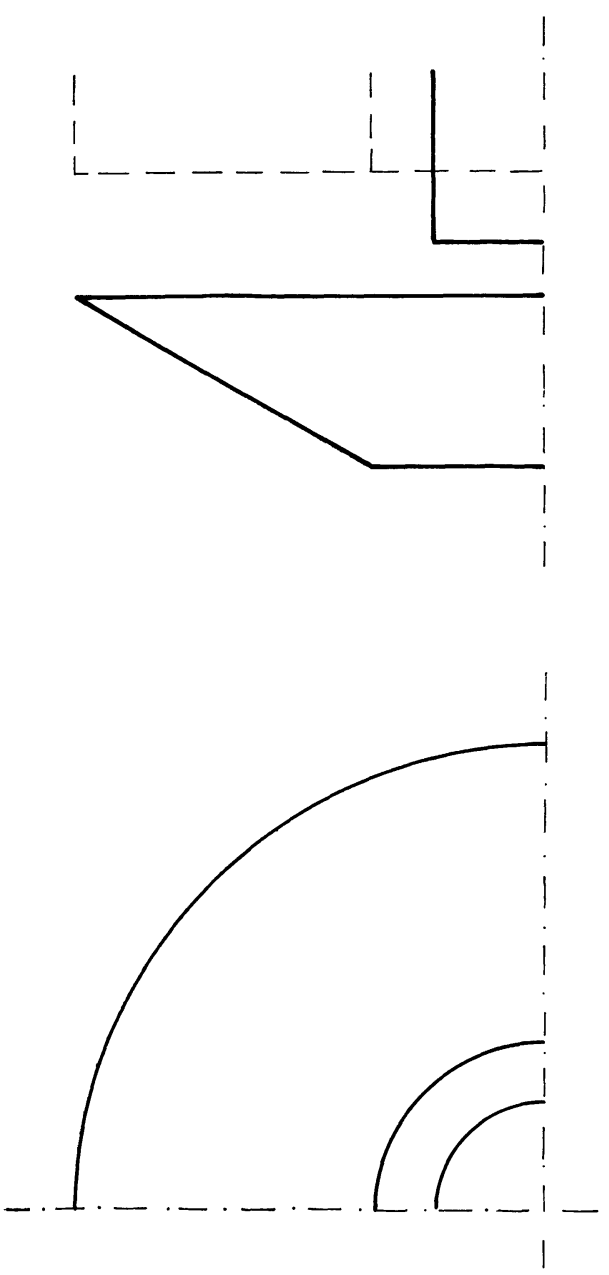

Fig. 4. - Miroir pour la détection des défauts transversaux dans un tube de révolution.

- sur la figure 5, $\alpha$ est donné par la relation (17). Le miroir peut être usiné par-une fraise dont l'axe, incliné de $45^{\circ}$, enveloppe un cylindre de rayon $R \sin i$ en $y$ décrivant une hélice de pas $2 \pi R \sin i$ [1].

5. Délimitation du miroir. - Le plan d'incidence $A M B$ fait un angle constant $\gamma$ avec $x O y$ et l'on a :

$$
\cos \gamma=\frac{\operatorname{tg} j}{\operatorname{tg} i}
$$

Si on considère une hélice $(\mathrm{H})$ tracée sur le cylindre (C) et telle que sa tangente fait précisément l'angle $\gamma$

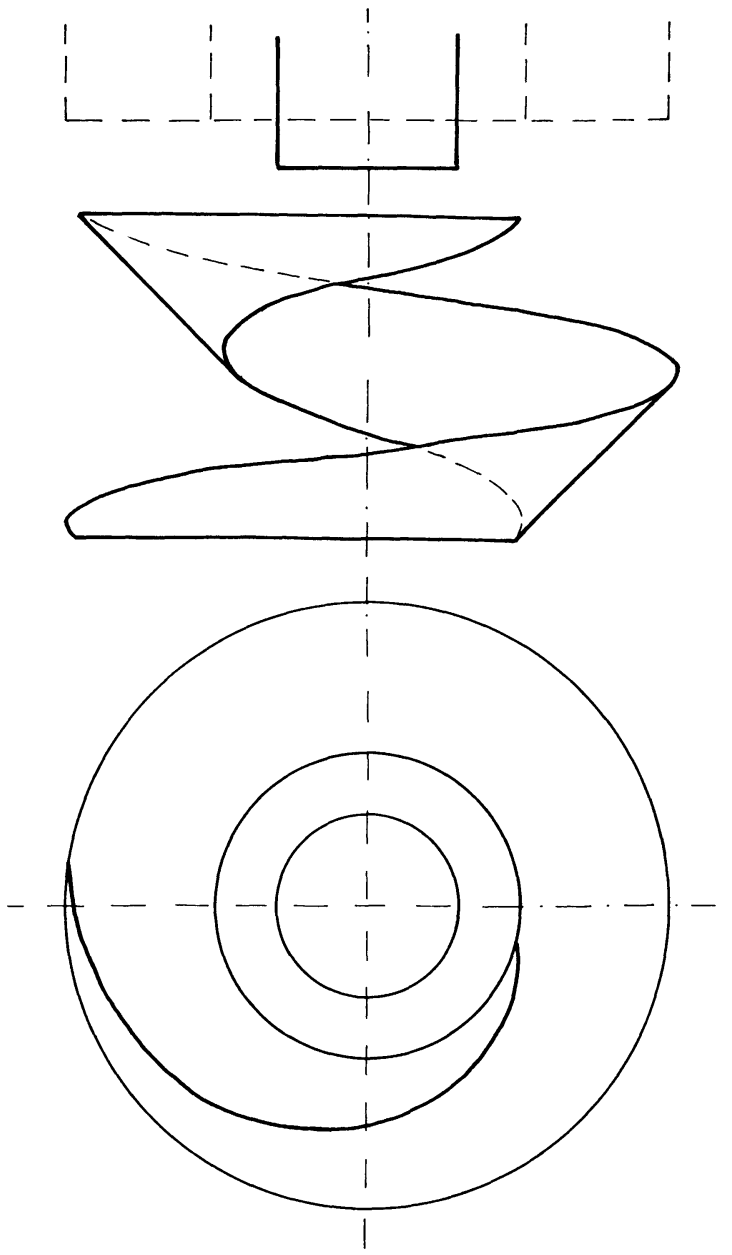

Fig. 5. - Miroir pour la détection des défauts longitudinaux dans un tube de révolution.

avec $x O y\left({ }^{2}\right)$, la vitesse de propagation le long de $(\mathrm{H})$ est identique pour les ondes incidentes et pour les ondes réfractées. Or, l'amplitude de l'onde réfractée est fonction de la longueur de l'arc de $(\mathrm{H})$ le long duquel est obtenue cette mise en phase. Pour que cette amplitude soit indépendante de la longitude du point examiné $Q$, il faut que la longueur de l'arc de (H) éclairé par le miroir soit indépendante de la longitude du point $A$, donc que la longueur de l'arc de la courbe $(\mathrm{K})$ tracé sur $(\mathrm{S})$ et éclairant l'arc de $(\mathrm{H})$ soit indépendante de la longitude du point $M$.

Cette courbe $(\mathrm{K})$ est intersection de (S) avec la surface réglée $(T)$ constituée par les rayons de la congruence des rayons incidents sur (C) qui passent par $(\mathrm{H})$. Une génératrice $M A$ de $(\mathrm{T})$ fait l'angle $2 \alpha$ avec $O z$ et est contenue dans le même plan $P M A$ qu'une génératrice (L) de (S). La courbe (K) est donc décrite par le point $M$ de (S) dont la projection $N$ sur $x O y$ est (Fig. 1) :

(2) (Un point $A$ de $(\mathrm{H})$ a pour coordonnées paramétriques :

$$
X=f(\varphi), \quad Y=g(\varphi), \quad Z=h(\varphi)
$$

en désignant par $h$ une fonction de $\varphi$ telle que

$$
h^{\prime}(\varphi)= \pm \operatorname{tg} \gamma \sqrt{\left.f^{\prime 2}(\varphi)+g^{\prime 2}(\varphi)\right)} .
$$


- sur la droite $G N$, d'éq. (4),

- telle que

$$
G N=M A \sin 2 \alpha
$$

on a :

$$
\overline{M A}=\overline{U A}=\overline{G A}-\overline{G U}
$$

d'où :

$$
\begin{aligned}
G N=[h(\varphi)-\operatorname{tg} \alpha[f(\varphi) \cos \psi+ \\
+g(\varphi) \sin \psi+\lambda(\psi)]] \sin 2 \alpha
\end{aligned}
$$

Pour que toutes les courbes $(\mathrm{K})$ que l'on peut tracer sur $(\mathrm{S})$ soient limitées à des arcs de même longueur, la surface (S) doit d'abord être comprise entre deux cylindres $\left(\Sigma_{1}\right)$ et $\left(\Sigma_{2}\right)$ dont la distance selon $G N$ est constante, tels que, par exemple :

$$
b_{1}<G N<b_{2}
$$

en désignant par $b_{1}$ et par $b_{2}$ deux constantes.

Seule est efficace la partie de la surface (S) qui est éclairée par la congruence des rayons parallèles émis par la source. Il reste donc à délimiter (S) par deux courbes $\left(K_{1}\right)$ et $\left(K_{2}\right)$ se déduisant l'une de l'autre par une translation d'un pas $p$ parallèlement à $O z$. De plus, on choisira $b_{2}-b_{1}<p \operatorname{cotg} \alpha$ afin que les rayons réfléchis par le miroir puissent atteindre (C) quand $p \neq 0$.

Ainsi, la zone éclairée sur le cylindre (C) est un parallélogramme curviligne délimité par deux arcs d'hélices $\left(\mathrm{H}_{1}\right)$ et $\left(\mathrm{H}_{2}\right)$ et par deux spires complètes d'hélices de pas $p$ (Fig. 6).

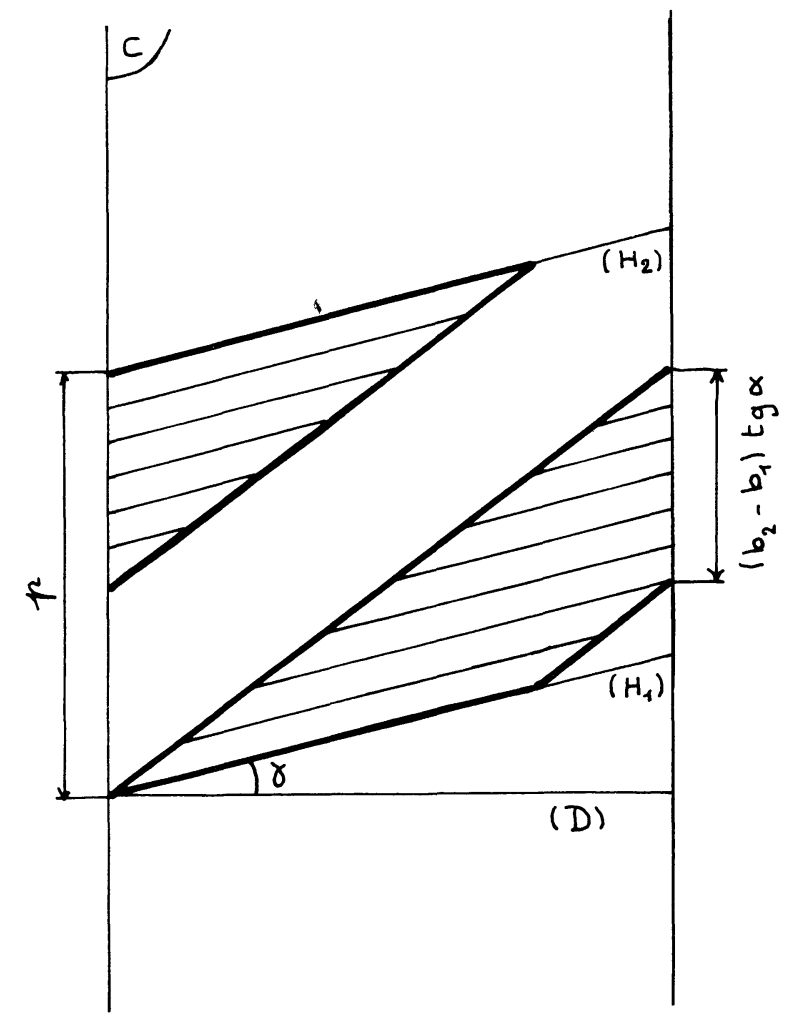

Fig. 6. - Représentation développée du cylindre (C) montrant la zone éclairée.
Lorsque $\alpha$ est donné par la relation (15) (cas par exemple des Fig. 2 et 4$)$, la courbe $(\mathrm{H})$ est une génératrice de $(\mathrm{C})$ et $(\mathrm{K})$ est une génératrice $(\mathrm{L})$ de $(\mathrm{S})$ située dans le plan méridien passant par $(\mathrm{H})$. Pour que la longueur du segment (L) soit indépendante de la longitude, on délimitera (S) par deux sections équatoriales.

Lorsque $\alpha$ est donné par la relation (17) (cas par exemple des Fig. 3 et 5), la courbe $(\mathrm{H})$ est une directrice de $(\mathrm{C})$ et $(\mathrm{K})$ est une section de (S) par le plan équatorial passant par $(\mathrm{H})$. Lorsque (C) est un cylindre de révolution (Fig. 5), (K) est une développante du cercle de rayon $R \sin i$. Pour que la longueur de l'arc de (K) soit indépendante de la longitude, on délimitera (S) par les sections avec deux cylindres coaxiaux avec (C).

6. Applications. - Cette étude a été faite dans le but d'engendrer des ondes ultrasonores dans un tube de révolution afin d'en contrôler la santé. Il entre, en effet, plusieurs centaines de kilomètres de tubes dans la construction d'une centrale nucléaire et un contrôle à $100 \%$ est indispensable pour les gaines du combustible et pour les tubes d'échangeur. Or, les spécifications imposent de plus en plus souvent que ces tubes soient contrôlés à la fois par ultrasons et par courants de Foucault. Ce dernier contrôle se fait simplement, et rapidement, par translation à travers une bobine encerclante alors que les ultrasons ont nécessité jusqu'ici une exploration hélicoïdale du tube, donc une mécanique lente et complexe. Il était tentant d'utiliser le même banc par translation pour effectuer les deux contrôles en ligne.

Nous avons vu plus haut comment devaient être définis les deux miroirs permettant de détecter l'un les défauts transversaux (Fig. 3) et l'autre les défauts longitudinaux (Fig. 5) sans mise en rotation des tubes. Nous avons placé ces miroirs de part et d'autre d'une bobine à courants de Foucault, chacun étant face à un traducteur ultrasonore annulaire plan, cet ensemble étant centré par deux bagues et suspendu dans une cuve d'eau à niveau constant [2].

Les essais ont porté sur plusieurs sortes de tubes minces de petits diamètres (inox et zircaloy). Les paramètres des miroirs ont été déterminés de façon à engendrer dans ces tubes des ondes de Lamb avec des traducteurs de $4 \mathrm{MHz}$. Dans le cas du miroir hélicoïdal, nous avons vérifié, sur un diagramme de Schlieren, que le rayonnement enveloppait bien le cylindre de rayon $R$ $\sin i(\grave{a} \pm 0,01 \mathrm{~mm})$. Nous avons effectivement obtenu, pour deux fentes externe et interne de mêmes dimensions usinées dans le tube, des échos différant de moins de $2 \mathrm{~dB}$, ce qui montre que la détection est indépendante de la profondeur à laquelle est situé le défaut. Pour vérifier qu'elle était également indépendante de la génératrice sur laquelle est situé le défaut, nous avons tourné les miroirs par rapport à l'ensemble tubetraducteurs : les variations sont inférieures à $1 \mathrm{~dB}$ pour le miroir conique et de l'ordre de $3 \mathrm{~dB}$ pour le miroir hélicoïdal. 
Ce dispositif permet de décupler la cadence de Il est possible que de tels miroirs trouvent égalemen) contrôle alors que l'investissement est bien moins coû- des applications en optique (cohérente en particulier) teux que pour les bancs classiques à défilement héli- ou en radio-électricité. coïdal.

\section{Bibliographie}

[1] Perdijon, J., Revue Phys. Appl. 9 (1974) 925.

[2] Perdijon, J., Rev. Métall. 72 (1975) 73. 